\title{
Seleção de Ferramenta CASE para o Estado da Bahia - Um caso de uso da NBR ISO/IEC 14102/1999
}

\author{
Maria Célia Furtado Rocha - PRODEB - Companhia de Processamento de Dados do Estado \\ da Bahia \\ Sara Raskin - CELEPAR - Companhia de Informática do Paraná
}

\begin{abstract}
Resumo
O presente artigo pretende analisar o processo de avaliação e seleção de ferramenta CASE para o Estado da Bahia, com o objetivo de identificar pontos relevantes da experiência e verificar as condições mais prováveis para a ocorrência de aplicações da NBR ISO/IEC 14102/1999 congruentes com as práticas preconizadas pelo novo modelo de gerenciamento. Palavras-chave: processo de avaliação; ferramenta CASE
\end{abstract}

\begin{abstract}
This paper is intended to analyse the "CASE-tools" selection in the Public Sector in Brazil. The case-study was conducted in the state of Bahia in a public committee for software selection and illustrates how norm applications may work in a quality management way.
\end{abstract}

Key-word: CASE tool selection

\section{Introdução}

A partir da década de 70, as empresas são desafiadas a capacitar-se para fazer face a novos e mais aguçados competidores num mercado cada vez mais globalizado. No final dos anos 80, técnicas de gerenciamento pela qualidade passam a receber especial atenção como forma de adequar a produção a exigências declaradas ou implícitas dos consumidores. A idéia é viabilizar a redução do custo dos produtos e flexibilizar a produção " $a$ fim de atender $a$ demandas de grupos específicos de consumidores" [5].

De um modo ou de outro, os princípios da qualidade disseminam-se, desde então, através de inúmeras práticas administrativas, que se caracterizam por maior integração entre membros da organização, criação de um ambiente mais participativo, tendência para a realização de trabalhos e solução de problemas em equipe - em detrimento de trabalhos baseados exclusivamente na ação individual.

O novo modelo de gerenciamento preconiza o desenvolvimento do autocontrole dos envolvidos e a conseqüente diminuição da necessidade de supervisão. Orienta-se a processos e focaliza a visão do cliente, assumindo um ponto de vista mais abrangente que a prática produtiva anterior, que privilegiava a idéia de qualidade do engenheiro [7]. Medição e análise factual dão suporte às avaliações, que devem ser permanentes, de modo a subsidiar necessárias correções de curso [7].

$\mathrm{O}$ gerenciamento pela qualidade enfatiza a necessidade de, primeiramente, se compreender o processo em uso na organização, propiciando a articulação do conhecimento entre aqueles que se encontram, discutem o assunto e passam a atuar a partir de um framework comum [7]. Como se vê, a abordagem da qualidade propugna novas maneiras de integrar a organização, tornando mais ágil sua adaptação às demandas do mercado e da sociedade, pelo acúmulo de capacitação tecnológica que propicia. 
Uma possibilidade de balizar o processo de gerenciamento pela qualidade em um nível global surge com o advento das normas internacionais ISO 9000, que começam a ser publicadas a partir de 1987, para tentar garantir a padronização dos requisitos de garantia de qualidade que um fornecedor deve atender, e que obtêm a adesão da comunidade internacional [6]. ${ }^{1}$

Do ponto de vista específico da produção de software no Brasil, um conjunto significativo de normas vem sendo publicado pela ABNT, através do Subcomitê de Software (SC 21:10). Essas normas referem-se a duas áreas: engenharia de software e bancos de dados [6]. O presente artigo discute a aplicação de uma norma produzida pela ISO - Organização Internacional para Normalização em conjunto com a IEC - Comissão Eletrotécnica Internacional, organizações que formam o sistema especializado para normalização mundial [1], traduzida pela ABNT e que se refere à primeira das áreas citadas.

O caso que aqui se expõe relata uma experiência na avaliação e seleção de ferramenta CASE para o Estado da Bahia, orientada pela NBR ISO/IEC 14102/1999. A experiência foi capitaneada pela PRODEB - Companhia de Processamento de Dados do Estado da Bahia, que convidou seus principais clientes a participarem da Comissão de Seleção.

O presente artigo pretende analisar a experiência, com o objetivo de identificar seus pontos relevantes e verificar as condições mais prováveis para a ocorrência de aplicações da NBR ISO/IEC 14102/1999 congruentes com as práticas preconizadas pelo novo modelo de gerenciamento.

Antes de relatar o caso e fazer ilações a respeito, far-se-á uma breve apresentação da NBR ISO/IEC 14102/1999.

\section{Orientação para Avaliação e Seleção de Ferramenta CASE}

Ferramentas CASE (Computer Aided System Engineering) são apontadas como elementos chave no controle de processos da engenharia de software. O processo de escolha de ferramentas é de grande importância e deve levar em consideração tanto requisitos técnicos quanto gerenciais. Possuir um método para selecionar as ferramentas mais apropriadas à organização, entender como elas interagem no processo de desenvolvimento de software, conhecer suas vantagens e limites, acompanhar tendências e assuntos discutidos internacionalmente é de grande importância para as empresas de software.

A NBR ISO/IEC 14102/1999 - Tecnologia de Informação - Orientação para Avaliação e Seleção de Ferramentas CASE descreve um conjunto de processos bem definidos para orientar organizações que visam automatizar o ciclo de vida do desenvolvimento e construir software de qualidade. Toma como referência o modelo de avaliação de produto de software, as características de qualidade de software e os processos do ciclo de vida do desenvolvimento, objeto de outras normas de qualidade de software. Esses processos são úteis tanto para os usuários quanto para os fornecedores de ferramentas CASE, e sua aplicação poderá levar a resultados mais efetivos para a organização que os adota.

Os processos e atividades estabelecidos pela norma são genéricos e devem ser adaptados de acordo com o contexto em que se dá a adoção da tecnologia. São eles: processo de iniciação; processo de estruturação; processo de avaliação e processo de seleção.

\footnotetext{
${ }^{1} \mathrm{Na}$ revisão de 2000, há um avanço nos objetivos de qualidade desta norma para um "modelo que visa alcançar a satisfação dos clientes" [6], que envolve mais do que os requisitos especificados pelos clientes.
} 
O processo de iniciação visa estabelecer objetivos gerais e requisitos da avaliação e seleção para definir aspectos de gerenciamento e dimensionar esforços necessários. É composto de três atividades: definição de metas; estabelecimento de critérios de seleção e planejamento do projeto.

O processo de estruturação desempenha um papel fundamental pois organiza os requisitos contra os quais as ferramentas candidatas serão avaliadas. Seu objetivo é elaborar um conjunto de requisitos estruturados e obter informações acerca das ferramentas. Informações e diretrizes da organização deverão estar disponíveis para serem utilizadas. Este processo é composto por: análise de requisitos; levantamento de informações sobre ferramenta CASE e identificação de ferramentas CASE candidatas a partir dos resultados das duas últimas atividades.

O objetivo do processo de avaliação é produzir relatórios técnicos que servirão de base ao processo de seleção, fornecendo um perfil das ferramentas avaliadas com relação à qualidade e a outras características. Este processo é composto das seguintes atividades: preparação para avaliação em que consolidam-se os detalhes da avaliação em um plano; avaliação das ferramentas CASE e relato da avaliação, em que se elabora relatório com os resultados da avaliação de cada ferramenta.

A proposta do processo de seleção é identificar a ferramenta CASE mais adequada entre as candidatas e certificar-se de que a ferramenta recomendada atenda aos objetivos originais. O processo de seleção compara os resultados de avaliação das ferramentas candidatas para determinar qual é a mais apropriada. É composto por quatro atividades: preparação para a seleção; aplicação aos resultados da avaliação; recomendação da decisão da seleção e validação da decisão da seleção contra os objetivos originais.

Além dos processos, a norma define um amplo espectro de aspectos a serem avaliados, organizados sucessivamente em características e subcaracterísticas relacionadas ao processo do ciclo de vida, ao uso da ferramenta, à qualidade, ao fornecedor e ao produto.

A NBR ISO/IEC 14102/1999 fornece, dessa maneira, um guia para um processo de avaliação e seleção bem planejado e organizado, para que se resguardem os investimentos da empresa, uma vez que a aquisição de ferramentas CASE e sua adoção não são atividades simples e requerem muita atenção no estabelecimento de critérios de seleção para que os objetivos sejam satisfeitos. O uso desse modelo de referência aumenta a confiabilidade da tomada de decisão, aumentando as chances de a organização alcançar os benefícios prometidos pela automatização do processo de software.

A seguir, apresenta-se um caso de aplicação da NBR ISO/IEC 14102/1999.

\section{O Processo de Seleção da Ferramenta CASE para o Estado da Bahia}

A motivação para o processo de avaliação e seleção de ferramenta CASE para o Estado da Bahia relaciona-se, em primeiro lugar, com a necessidade, identificada pela PRODEB, de implantar a administração de dados no âmbito do Estado e, em segundo lugar, com a necessidade de dar suporte ao desenvolvimento de sistemas realizados em suas várias unidades [3]. Os passos dados durante esse processo são relatados a seguir.

Inicialmente, realizaram-se o reconhecimento das principais ferramentas existentes no mercado e a investigação de suas principais características, através de contato direto com fornecedores, demonstrações de aplicativos e folhetos de divulgação comercial. Em seguida, encaminhou-se um plano de ação à direção da companhia, no qual delimitaram-se 5 fases do 
processo de seleção - Instalação da Comissão, Conhecimento dos Produtos, Validação dos Critérios de Seleção, Seleção de Ferramenta, Elaboração de Relatório Final - com a estimativa de custo máximo para 12 cópias do software.

A partir da concordância com os objetivos propostos - prover o gerenciamento do acervo de dados do Estado da Bahia; manter a qualidade das bases de dados e aumentar a eficiência do processo de desenvolvimento de sistemas - e da aprovação do plano pela direção, tratou-se de levantar os requisitos básicos que a ferramenta deveria atender. Este levantamento foi orientado pelo conhecimento obtido durante a investigação inicial das ferramentas oferecidas no mercado e baseou-se na experiência dos técnicos da PRODEB em administração de dados e em desenvolvimento de sistemas. Conhecimentos de rede também foram necessários para se estabelecerem as configurações mais desejáveis para suportar a arquitetura da administração de dados proposta, que prevê a existência de administração centralizada das entidades de uso comum ao Estado, cuja atualização é de responsabilidade de determinados órgãos.

Houve ainda a preocupação em levantar informações acerca de outros processos de seleção que empresas similares porventura tivessem experimentado em todo o Brasil. Em contato com a CELEPAR - Companhia de Informática do Paraná, a PRODEB soube da existência da NBR ISO/IEC 14102/1999, votada e ainda não publicada naquele momento. Quase imediatamente essas companhias estabeleceram um acordo de intercâmbio técnico que permitiu a visita da técnica da CELEPAR responsável por coordenar a Comissão de Estudos CE 21:101.05, de Ferramentas e Ambiente, que compõe o Subcomitê de Software da ABNT, sediado em Curitiba.

Durante três dias, a técnica da CELEPAR apresentou os processos previstos na norma e tirou dúvidas dos técnicos da PRODEB que encontravam grande dificuldade em interpretar a descrição dos aspectos associados às características e subcaracterísticas sugeridos para a avaliação das ferramentas, provavelmente em decorrência da tradução da norma que, muitas vezes, tornou o texto hermético ou ambíguo.

Além disso, a técnica esclareceu que o processo de avaliação não poderia realizar-se a partir da simples escolha das características a serem avaliadas, mas deveria envolver um detalhamento das mesmas até o ponto em que fossem refletidas em atributos mensuráveis, o que diminuiria sensivelmente a subjetividade do processo. Nesse momento ficou explícita uma lacuna de conhecimento do grupo, cujos integrantes jamais haviam experimentado, anteriormente, processos de avaliação norteados por modelos de avaliação de qualidade de software.

Assim, pode-se dizer que houve uma fase preparatória, que durou 4 meses (janeiro a abril de 2000), envolvendo atividades consistentes com os processos de iniciação e estruturação previstos na norma, na qual técnicos da PRODEB pertencentes a distintos setores - Banco de Dados, Administração de Dados, Desenvolvimento, Normas e Padrões de Desenvolvimento - fizeram um grande esforço para absorver as prescrições da norma e adaptar requisitos levantados à ampla gama de características propostas na mesma ${ }^{2}$. A NBR 14102 cumpriu o papel de organizadora de conhecimentos prévios, orientou os processos que viriam a se realizar e funcionou como um check list para o levantamento de requisitos técnicos e comerciais, já que relaciona características do produto e do produtor que não podem ser ignoradas em avaliações de produtos dessa natureza.

\footnotetext{
${ }^{2}$ A adaptação da norma incluiu supressão, mas também adição de características a serem avaliadas.
} 
Finalmente, a Comissão de Seleção de Ferramenta CASE foi instituída em maio de 2000, tendo sido composta pela própria PRODEB e por 6 Secretarias de Estado - de Administração, Fazenda, Planejamento, Saúde, Educação e Segurança Pública -, para as quais foi repassado o conhecimento adquirido na fase preparatória e submetido o elenco de características e subcaracterísticas a serem avaliadas, tipificadas em desejáveis e obrigatórias, juntamente com seus respectivos níveis de importância, que obedeceram à seguinte escala: 1 Baixo, 2 - Regular, 3 - Bom e 4 - Alto.

Os quatro grandes grupos de características avaliadas receberam os seguintes níveis de importância: Processos do Ciclo de Vida e Uso da Ferramenta CASE, nível 4; Características de Qualidade e Dados do Fornecedor e do Produto, nível 3.

Uma vez realizados os ajustes propostos pela Comissão, esta buscou estabelecer o perfil das ferramentas avaliadas para subsidiar a seleção, de maneira coerente com processo de avaliação previsto na norma.

O Plano de Avaliação previu duas fases:

1. Coleta de informações junto aos fornecedores, através de questionário, para se verificar a capacidade de as ferramentas candidatas atenderem a todas as características (desejáveis e obrigatórias).

2. Demonstração, pelos fornecedores, da facilidade de implementar as características mais importantes para a consecução das metas e objetivos.

Em maio de 2000, o questionário correspondente à primeira fase da avaliação foi enviado para fornecedores de três diferentes ferramentas CASE disponíveis no mercado, às quais se teve acesso. São elas: System Architect; a solução oferecida pela Computer Associated, que inclui o produto ErWin, e a ferramenta Oracle Designer.

Com base nas respostas ao questionário, computou-se valor 1 para cada característica atendida pela ferramenta. Em alguns casos, pontuou-se, em ordem crescente, de 1 a 3, segundo sua maior capacidade de operar com o maior número de tabelas e de usuários acessando simultaneamente o repositório e segundo o menor custo de licenças de uso, treinamento e horas de consultoria. O somatório dos pontos obtidos para cada conjunto de subcaracterísticas e características foi sucessivamente multiplicado pelo nível de importância a ele atribuído. Obteve-se, assim, uma primeira pontuação para as ferramentas em análise.

Em junho daquele ano, os fornecedores apresentaram as ferramentas, utilizando um modelo de dados simplificado, elaborado pela Comissão de Seleção, que simulava algumas situações críticas para o gerenciamento e garantia da qualidade do acervo de dados do Estado. A apresentação seguiu roteiro predefinido, foi filmada e aberta a técnicos e a todos os fornecedores interessados.

Cada avaliador atribuiu valor de 1 a 4 (1 - Ruim; 2 - Regular; 3 - Bom e 4 - Muito Bom) a cada uma das características apresentadas pelos fornecedores. Em seguida, calculouse a média aritmética dos pontos obtidos por cada ferramenta, multiplicada, em seguida, pelo nível de importância atribuído às subcaracterísticas e características.

A Comissão de Seleção reuniu-se, posteriormente, para interpretar os resultados, quando se nivelou o entendimento das facilidades apresentadas e se revisaram os valores a elas atribuídos. $\mathrm{Na}$ ocasião, os técnicos especialistas em rede convidados para assistirem as apresentações deram seus pareceres sobre o grau de adequação demonstrado pelas ferramentas ao ambiente físico de operação. Houve itens cuja demonstração foi tida como insatisfatória pela Comissão. Neste caso, ou foram desconsiderados ou solicitou-se aos 
fornecedores atestarem formalmente que as ferramentas permitiam a realização das operações envolvidas.

As pontuações obtidas foram analisadas criticamente pela comissão, tendo em vista os objetivos perseguidos. Essa reflexão, recomendada pela norma, é fundamental para garantir a aderência da escolha à orientação original, evitando desvios promovidos por um olhar eminentemente tecnicista em detrimento das necessidades do negócio, que motivaram o processo.

Para efeitos de pontuação final, atribuiu-se peso 3 aos pontos obtidos por cada ferramenta a partir das informações prestadas pelos fornecedores via questionário, e peso 7 para os pontos obtidos na demonstração. Essa distribuição de pesos reflete a convicção de que o grande diferencial entre as ferramentas encontra-se no modo como cada uma implementa as características avaliadas: a forma como o fazem torna seus processos mais ou menos amigáveis ao usuário e seus resultados mais ou menos confiáveis.

A ferramenta Oracle Designer obteve pontuação máxima no processo de avaliação e foi recomendada para a seleção. A Comissão de Seleção de Ferramenta CASE aceitou esta recomendação, por considerar ser a ferramenta Oracle Designer a que melhor atende às metas e diretrizes que orientaram o processo de seleção.

\section{Comentários Sobre o Caso}

A importância da realização do processo de avaliação e seleção de ferramenta CASE para o Estado da Bahia de maneira sistemática justifica-se já na apresentação do Relatório Final da Seleção, que diz:

É cada vez mais importante, para o setor público, capacitar-se para exercer de maneira eficaz a seleção de tecnologias da informação. A princípio, a tarefa pode parecer fácil, mas, frente a um mercado que oferece inúmeras opções, é preciso traçar objetivos muito claros, seja qual for o nível previsto para o uso da tecnologia - operacional, gerencial ou estratégico. Isto requer uma série de conhecimentos: da tecnologia em si mesma, das estratégias e metas a serem alcançadas. [3]

Como o relato do caso mostrou, a primeira providência será determinar claramente os objetivos da avaliação e seleção, sem o quê não se poderão estabelecer critérios consistentes para a tomada de decisão.

Verifica-se que manter o sentido de finalidade é fundamental para evitar desvios provenientes das restrições impostas por conhecimentos sempre fragmentados e submetidos à experiência prévia dos envolvidos [4]. Por esse motivo os objetivos devem ser, sempre que possível, retomados explicitamente. É o que propõe a NBR 14102, que estabelece atividade de validação na qual os resultados da avaliação deverão ser confrontados com os objetivos iniciais.

Pode-se dizer que o caso aqui relatado orientou-se pela busca da racionalidade, que tem impacto direto sobre os seus resultados, mas também pela legitimação do processo, conforme exposto no Relatório Final: "Para tornar o processo de avaliação e seleção imparcial, objetivo e repetitivel, optou-se por adaptar a NBR ISO/IEC 14102/1999 [...]. Para torná-lo transparente, criou-se uma Comissão de Seleção" [3].

Se chegou a realizar esses objetivos é uma questão que foi parcialmente investigada em pesquisa conduzida, em 2000, pelo Núcleo de Pós-Graduação da Escola de Administração da Universidade Federal da Bahia, destinada a levantar os principais fatores potencializadores e restritivos da aprendizagem verificada no processo e que envolveu 9 dos 10 membros da Comissão de Seleção. Sete dos 9 entrevistados consideraram que os critérios de avaliação 
utilizados adequavam-se aos objetivos fixados para o trabalho do grupo [2]. A pesquisa realizada demonstrou, ainda, a importância atribuída à metodologia como o principal recurso estratégico disponibilizado para a seleção da ferramenta, na qual se inclui o uso da norma, seguida por recursos de infra-estrutura e recursos humanos. O mapeamento das capacitações necessárias para desempenhar o papel na Comissão de Seleção apontou que o grupo deveria reunir, além de conhecimentos técnicos na área de informática, conhecimentos sobre "organização de processos de seleção, metodologia de análise, Lei Estadual de Licitação e visão do negócio governo" [2]. Finalmente, os resultados da pesquisa mostram o quanto é vital investir na criação de um clima favorável à troca de conhecimentos entre os participantes do processo, possibilitando, desse modo, a construção conjunta do entendimento que orientará a atuação da equipe a partir de um framework comum, consistente com a ação organizacional.

As leituras da experiência do Estado da Bahia mostram aspectos que devem ser considerados em processos de avaliação e seleção de ferramenta CASE, apontados a seguir.

\section{Considerações Finais}

O relato do caso indica a existência de condições mais favoráveis para o sucesso de processos de avaliação e seleção de produtos de software orientados por normas de qualidade de software em geral e pela NBR ISO/IEC 14102/1999 em particular.

Inicialmente verifica-se ser desejável o conhecimento prévio de processos de avaliação de produtos realizados com base em normas de qualidade de software. A correta compreensão dos procedimentos e do nível de detalhamento necessário ao julgamento das características do produto só será alcançada mediante forte apoio daqueles familiarizados com processos similares. Isto ficou provado pelas dificuldades encontradas pelos membros da Comissão de Seleção para alavancar a compreensão de pontos focais da norma, que inclui o processo de estruturação e o processo de seleção propriamente dito.

Houve ainda dificuldade em interpretar as características elencadas pela norma, cuja tradução nem sempre alcançou os melhores resultados. A sua leitura demandou longo tempo, também em razão da multiplicidade de capacitações e de pontos de vista que um processo deste tipo e com esta abrangência normalmente envolve. Sendo assim, é recomendável estabelecer um momento inicial de entendimento, com ênfase nos objetivos da seleção e que envolva a compreensão da norma. Ainda que prolongado, esse passo tem o objetivo de assegurar o compartilhamento de experiências, o ajuste de pontos de vista e a construção de um framework comum indispensável para assegurar a qualidade do processo e dos resultados alcançados.

Diante de um grande elenco de características a serem avaliadas, é fundamental estabelecer níveis de importância para cada uma delas, partindo dos grandes agrupamentos até chegar a subcaracterísticas atômicas. ${ }^{3}$ Essa orientação visa manter a coerência entre a atribuição de importância e os objetivos originais da seleção.

Da mesma forma, o plano de avaliação deve enfocar aspectos relevantes, prevendo demonstrações de acordo com modelos reais e abrangentes das funcionalidades requeridas.

Finalmente, a ampla discussão dos aspectos observados pelos avaliadores é uma prática desejável para se tentar minimizar mal entendidos e afinar visões, constituindo-se uma

\footnotetext{
${ }^{3}$ Correspondem à menor subdivisão das características que compõem categorias de avaliação superiores [1] e que se constituem em aspectos tangíveis passíveis de serem pontuados durante a avaliação das ferramentas.
} 
validação imprescindível do processo realizado. Esta prática é coerente com a noção de racionalidade limitada (satisfacing) defendida por Simon que diz ser a mente humana incapaz "de aplicar a uma decisão todos os aspectos de valor, conhecimento e comportamento que poderiam ter importância" [4], representando a decisão apenas "a melhor solução encontrada naquelas circunstâncias" [4].

O relato da experiência demonstra que a NBR ISO/IEC 14102/1999 orienta e propicia a constituição de um ambiente mais participativo, favorecendo a troca de experiências e a articulação do conhecimento detido pelos membros da organização. Estes, quando submetidos a um clima favorável, passam a construir coletivamente um novo entendimento acerca das possibilidades de uso da ferramenta CASE, mas também passam a acreditar na possibilidade de práticas mais participativas de trabalho conjunto. Assim, é possível que o uso da norma auxilie a conduzir aspectos centrais do novo modelo de gerenciamento, que devem ser garantidos no processo.

Em suma, o uso da norma potencializou a capacidade de aprender e de socializar o produto desse aprendizado entre membros da Comissão de Seleção e até mesmo entre membros das empresas congêneres PRODEB e CELEPAR, com fomento do acúmulo de capacitação tecnológica e previsíveis benefícios para o Estado da Bahia, que optou por um processo de escolha orientado a maior racionalização e transparência.

\section{Referências}

[1] ABNT. NBR ISO/IEC 14102/1999. Tecnologia de Informação - Orientação para Avaliação e Seleção de Ferramentas CASE. Rio de Janeiro: ABNT, 1999. mimeo.

[2] LOIOLA, Elizabeth; ROCHA, Maria Célia F. Aprendizagem no Processo de Seleção de Ferramenta CASE para o Estado da Bahia. In: ENANPAD2001, 25, 2001. Anais... Campinas: ANPAD, 2001. p. 1-15.

[3] PRODEB. Seleção de Ferramenta CASE para o Estado da Bahia. Relatório Final. Salvador, setembro de 2000.

[4] SIMON, Herbert Alexander. Comportamento administrativo; estudo dos processos decisórios nas organizações administrativas. Tradução de Aluizio Loureiro Pinto. 2 ed. rev. RJ: FGV, 1970.

[5] VIEIRA, Marcelo Milano F.; CARVALHO, Cristina Amélia P. de. Qualidade e Objetivos: Implicações Teóricas e Metodológicas para a Análise das Organizações. In: VIEIRA, Marcelo Milano F.; OLIVEIRA, Lúcia Maria B. de. (orgs.). Administração Contemporânea. Perspectivas Estratégicas. São Paulo: Atlas, 1999. p. 121-147.

[6] WEBER, KIVAL C.; ROCHA, Ana Regina C. da; NASCIMENTO, Célia J. do. Qualidade e Produtividade em Software. 4 ed. São Paulo: Makron Books, 2001.

[7] WINTER, Sidney G. 'Organizing for Continuous Improvement. Evolutionary Theory Meets the Quality Revolution'. In: COHEN, Michael D.; SPROULL, Lee S. (eds.) Organizational Learning (Organization Science). California, London: Sage Production Ed., 1996. p. 460-483. 